\title{
Natura@economía
}

ISSN 2226-9479 (Versión electrónica)

Website: http://revistas.lamolina.edu.pe/index.php/neu

ARTÍCULO ORIGINAL

\section{Mercados para la conservación de bosques en Perú: una crítica desde la economia}

\section{Markets forest conservation in Peru: A critique from the economics}

\author{
Miguel Ángel La Rosa Salazar'; Carlos Minaya ${ }^{1}$; Luis Guillén ${ }^{1 *}$ \\ ${ }^{1}$ Universidad Nacional Agraria La Molina, Lima, Perú. Email: lguillen@lamolina.edu.pe
}

Recepción: 30/06/2019; Aceptación: 15/12/2020

\begin{abstract}
Resumen
El objetivo del presente estudio fue analizar las deficiencias en la implementación de MRSE, a partir de dos distintas perspectivas teóricas de la economía, ambiental e institucional, considerando la dinámica de la deforestación en la Amazonía peruana, en base a un modelo de competencia de rentas. Se determinó que las características de los servicios ecosistémicos no incentivan la aparición de mercados per se, $\mathrm{y}$, por ende, estos deben ser incentivados externamente, implicando costos de transacción considerablemente altos. El estudio concluye que es necesario hacer una revisión de la definición de los MRSE en relación con la conservación de los bosques, apoyándose en la teoría institucional para acoger las diversas formas de gobernanza que podrían aparecer sobre estos mecanismos.
\end{abstract}

Palabras clave: servicios ecosistémicos; deforestación; economía ambiental; economía institucional; costos de transacción; estructuras de gobernanza.

\begin{abstract}
The objective to the study was analyze shortcomings in the design and implementation of MRSE from two different economics theoretical perspectives, environmental and institutional, considering deforestation dynamics in the Peruvian Amazon based on a model of rents competition. It was determined that characteristics of ecosystem services do not encourage the emergence of markets per se, and therefore, it should be externally incentivized, meaning considerably high transaction costs. The study concludes that it is necessary to review the definition of MRSE in relation to forest conservation, relying on institutional theory to accommodate the various forms of governance that could appear on these mechanisms.
\end{abstract}

Keywords: ecosystem services; deforestation; environmental economics; institutional economics; transaction costs; governance structures.

Forma de citar el artículo: La Rosa, L.; Minaya, C.; Guillén, L. 2020. Mercados para la conservación de bosques en Perú: una crítica desde la economía. Natura@economía 5(2): 144-157. http://dx.doi.org/10.21704/ne.v5i2.1624 


\section{Introducción}

Entre los años 2002 y 2019, Perú perdió 2,00 Mha de bosque primario húmedo, lo que representó el $66 \%$ de su pérdida total de cobertura forestal en el mismo período. El área total de bosque primario húmedo en Perú disminuyó 2,80\% en este período (Global Forest Watch, 2020). La deforestación ocurre principalmente en bosques amazónicos de gran biodiversidad, representan el $95 \%$ de la cobertura forestal total del país (MINAG y MINAM, 2011).

La deforestación en el Perú se relaciona principalmente con las actividades agrícolas en la Amazonía. La evidencia basada en imágenes satelitales muestra que la agricultura es el principal driver de deforestación en el Perú (Curtis et al., 2018; Finer y Mamani, 2020, Finer et al., 2018; Vijay et al., 2018; Kalamandeen et al., 2018); mientras que, la minería aurífera aluvial ha ganado relevancia en los últimos años (Finer y Mamani, 2018). Esta evidencia ha sido corroborada a partir de estudios económicos en los que se encuentra una relación entre diversas variables de las actividades agrícolas y la deforestación en las regiones amazónicas peruanas (Lucich et al., 2015; Zegarra y Gayoso, 2015; La Rosa Salazar, 2016).

Para hacer frente a la deforestación, el gobierno peruano ha venido realizando algunos esfuerzos sobre la gestión de los bosques públicos, incorporando explícitamente la deforestación en la agenda nacional. Dichos esfuerzos incluyen varios cambios en instituciones $\mathrm{y}$ organizaciones forestales. SERFOR (Servicio Nacional Forestal y de Fauna Silvestre) desde 2013. La Ley Forestal y de Fauna Silvestre promulgada (Ley $N^{\circ} 29763$ ) y la primera Política Nacional Forestal y de Fauna Silvestre (Decreto Supremo $\mathrm{N}^{\circ}$ 009-2013-MINAGRI) Además, el 2010, el MINAM incluyó la deforestación como punto clave en la política ambiental (MINAM, 2011) y en la elaboración de la estrategia nacional sobre cambio climático (MINAM, 2014) Asimismo, el Acuerdo de París, considera la deforestación como un punto esencial (Pulgar - Vidal, 2016).

En los últimos años, el MINAM ha venido promoviendo fuertemente la implementación de esquemas de Mecanismos de Retribución por Servicios Ecosistémicos (MRSE) dentro de este marco. En 2014 se promulgó la Ley de MRSE (Ley N $\mathrm{N}^{\circ}$ 30215) cuyo reglamento (Decreto Supremo N 009-2016-MINAM) fue publicado en 2016. Si bien estos mecanismos aún presentan un desarrollo insipiente en el Perú (Llerena y Yalle, 2014; Quintero y Pareja, 2015)

Los MRSE son la respuesta a la búsqueda de la conservación de los ecosistemas. Detrás de ellos está el principio de que "la naturaleza se degrada allá donde la destrucción genera para su dueño más beneficio económico que la conservación, aunque la sociedad en su conjunto salga perdiendo" (Hajek y Martínez de Anguita, 2012). Los MRSE pueden contribuir en la conservación de ecosistemas al permitir que aquellos que los protegen se beneficien por las actividades que dejar de realizar en pro de esa protección.

En el caso de los bosques, la idea central es lograr que los usos forestales sostenibles sean más atractivos para los agentes económicos mediante la mercantilización de sus SE y su inclusión en la toma de decisiones sobre el uso de la tierra. Esto debe llevar a que los usos forestales sostenibles, que generalmente son menos rentables que otras actividades no sostenibles, como la agricultura y ganadería, sean de mayor interés para estos agentes (Van Hecken y Bastiaensen, 2010). Esto no necesariamente evita la deforestación. Sin embargo, puede ayudar a limitarla dependiendo de los valores mercantiles que se logren para los SE. 
Según Goldman y Thallis (2009), los MRSE han cobrado relevancia debido a la insuficiencia de las áreas naturales para proteger los ecosistemas en peligro. Esto se debe a tres causas: (i) estas áreas son, relativamente, pocas y pequeñas, muy aisladas y están muy sujetas a cambios, lo cual dificulta mantener extensiones lo suficientemente grandes como para permitir preservar las funciones de los ecosistemas y los flujos de SE; (ii) el cambio climático puede generar cambios drásticos en estas y otras áreas, por lo que el rango de especies y hábitats a proteger puede hacer insuficiente las capacidades de gestión; y (iii) conforme la población mundial se incremente, restringir el uso de la tierra con el solo objetivo de conservación es una forma precaria $\mathrm{y} / \mathrm{o}$ inviable de preservar el capital natural y los SE. Bruner et al. (2001) concuerdan en este último punto.

Si bien los MRSE presentan características prometedoras, su implementación depende de la creación de estructuras de gobernanza que aseguren la instalación de los mecanismos y su sostenibilidad. Desde la perspectiva de la economía institucional (sobre las reglas que estructuran comportamiento de agentes en la economía), el adecuado diseño de estas estructuras es fundamental. Esto se debe a que las estructuras de gobernanza implementadas interactuarán con otras instituciones preexistentes que, de ser incompatibles, pueden reducir la eficacia de los MRSE, incrementando los costos de transacción del funcionamiento de los mecanismos (Vatn, 2010). Esto significa la necesidad de realizar análisis profundos de la realidad en la que los MRSE se instalan, con la finalidad de entender la forma ideal que deben tomar las estructuras de gobernanza. Cabe señalar que, incluso cuando comúnmente el asunto institucional no se presenta en la definición de estos mecanismos, es un aspecto relevante en diferentes estudios globales (Wunder, 2005; Wunder et al., 2008) y nacionales (Llerena y Yalle, 2014; Quintero y Pareja, 2015) en los que los costos de transacción son una de las principales preocupaciones.

Este artículo tiene por objetivo analizar la debilidad en el proceso de implementación de MRSE en el Perú a partir de dos distintas perspectivas teóricas de la economía: ambiental e institucional. Esto se realiza para el caso de la conservación de los bosques Amazónicos peruanos, haciendo énfasis en el potencial de los MRSE para detener la expansión agrícola.

\section{Materiales y métodos}

Se realizó la revisión de literatura de dos diferentes ramas de la economía: ambiental e institucional. Se analizó la información obtenida para acercarla al caso peruano, se revisó información referente a cómo los MRSE podrían favorecer la conservación de los bosques en el Perú. Esto se hizo a través de la comparación de la información disponible sobre deforestación y producción agrícola en la Amazonía peruana. Finalmente, se revisó la información disponible sobre el establecimiento de los MRSE en el Perú.

\section{Resultados y discusión}

\section{Los MRSE para la conservación en el Perú}

Los MRSE surgen como enfoque de conservación debido a que parte significativa de los beneficios económicos de los SE no son tangibles y valorados por la sociedad, pues las externalidades positivas no pasan por el sistema de precios, y el carácter de bienes públicos de estos SE hacen que la cantidad (y calidad) de ellos sean inferiores a una asignación socialmente deseable (Hajek y Martínez de Anguita, 2012). Los SE son aquellos beneficios que las personas reciben a partir de las interacciones complejas de los ecosistemas (MEA, 2005). 
Entre los cuatro tipos de SE (Tabla 1) tan solo los servicios de provisión se relacionan estrechamente con los mercados. Ellos son comercializados por diversas industrias. Esto no sucede con los otros tipos de SE (de regulación, de hábitat, y culturales y de recreación). Para ellos, la formación de mercados no ocurre "espontáneamente". En sus casos, es necesario que terceros con ciertos intereses trabajen para establecer mecanismos, como es el caso de los mercados de carbono y los MRSE.

Tabla 1. Los tipos de servicios ecosistémicos

\begin{tabular}{|c|c|c|}
\hline Tipo servicio & Definición & Ejemplo \\
\hline de provisión & $\begin{array}{c}\text { Productos } \\
\text { obtenidos de } \\
\text { los ecosistemas }\end{array}$ & $\begin{array}{c}\text { Madera, } \\
\text { frutas, hierbas, } \\
\text { oxígeno, entre } \\
\text { otros }\end{array}$ \\
\hline de regulación & $\begin{array}{l}\text { Beneficios } \\
\text { obtenidos de } \\
\text { los procesos de } \\
\text { regulación de } \\
\text { los ecosistemas }\end{array}$ & $\begin{array}{l}\text { Ciclo del agua } \\
\text { y regulación } \\
\text { climática, } \\
\text { polinización, } \\
\text { control } \\
\text { biológico, } \\
\text { secuestro de } \\
\text { carbón, entre } \\
\text { otros }\end{array}$ \\
\hline de hábitat & $\begin{array}{l}\text { Basada en la } \\
\text { provisión de } \\
\text { otros servicios } \\
\text { ecosistémicos }\end{array}$ & $\begin{array}{l}\text { Mantenimiento } \\
\text { del ciclo de vida } \\
\text { y biodiversidad. }\end{array}$ \\
\hline $\begin{array}{l}\text { culturales y } \\
\text { de recreación }\end{array}$ & $\begin{array}{c}\text { Beneficios } \\
\text { intangibles } \\
\text { proveidos por } \\
\text { los ecosistemas }\end{array}$ & $\begin{array}{c}\text { Paisajes, hogar } \\
\text { de pueblos } \\
\text { originarios, } \\
\text { entre otros }\end{array}$ \\
\hline
\end{tabular}
Fuente: TEEB (2010), MINAG y MINAM (2011).

Los MRSE convocan a criterios de eficiencia económica y mecanismos redistributivos en el contexto de la declinación de SE, debido a la intervención humana. De esa manera, los MRSE surgen como una transferencia económica para compensar los costos de oportunidad de los proveedores de externalidades positivas (Smith, 2006; Wolcott, 2006; Muradian y Kumar, 2009). No obstante, para el caso específico de los bosques, se busca hacer que los beneficios que proveen a las personas sean considerados en la toma de decisiones. Estos beneficios pueden ser locales, regionales/nacionales y globales (Hanley et al., 2001).

La definición oficial de los MRSE, para el caso peruano, la provee el MINAM (2016a): "son los esquemas, herramientas, instrumentos e incentivos para generar, canalizar, transferir e invertir recursos económicos, financieros y no financieros, donde se establece un acuerdo entre contribuyente(s) y retribuyente(s) al servicio ecosistémico, orientado a la conservación, recuperación y uso sostenible de las fuentes de los servicios ecosistémicos. Un MRSE puede ser diseñado en base a uno o más servicios ecosistémicos". En este sentido, los principales puntos que deben estar contenidos en acuerdos de MRSE son (i) la ubicación y descripción del área del ecosistema donde se implementará el MRSE, (ii) las acciones específicas a las que se comprometen los contribuyentes, iii) la identificación de los servicios ecosistémicos, en tanto puedan ser estos beneficios sociales, ambientales y/o económicos; y iv) las acciones específicas para el monitoreo del cumplimiento del acuerdo.

\section{Modelo de competencia de rentas y MRSE}

Las rentas agrícolas y forestales compiten por prevalecer y determinar el uso de ciertas superficies de cobertura forestal. Angelsen (2010) explica que, las rentas agrícolas $\mathrm{y}$ forestales $\left(\mathrm{R}^{\mathrm{a}} \mathrm{y} \mathrm{r}^{\mathrm{f}}\right.$, respectivamente) dependen de los mismos factores: precios (p), nivel de producción (y), factores de producción $(1, \mathrm{k})$ y sus costos unitarios $(\mathrm{w}, \mathrm{q})$. Adicionalmente se considera el costo de transporte (v), asociado a la distancia hacia el mercado final de destino (d). Cómo interactúan $\mathrm{R}^{\mathrm{a}}$ y $\mathrm{R}^{\mathrm{f}}$ se muestra en la Figura 1 . 


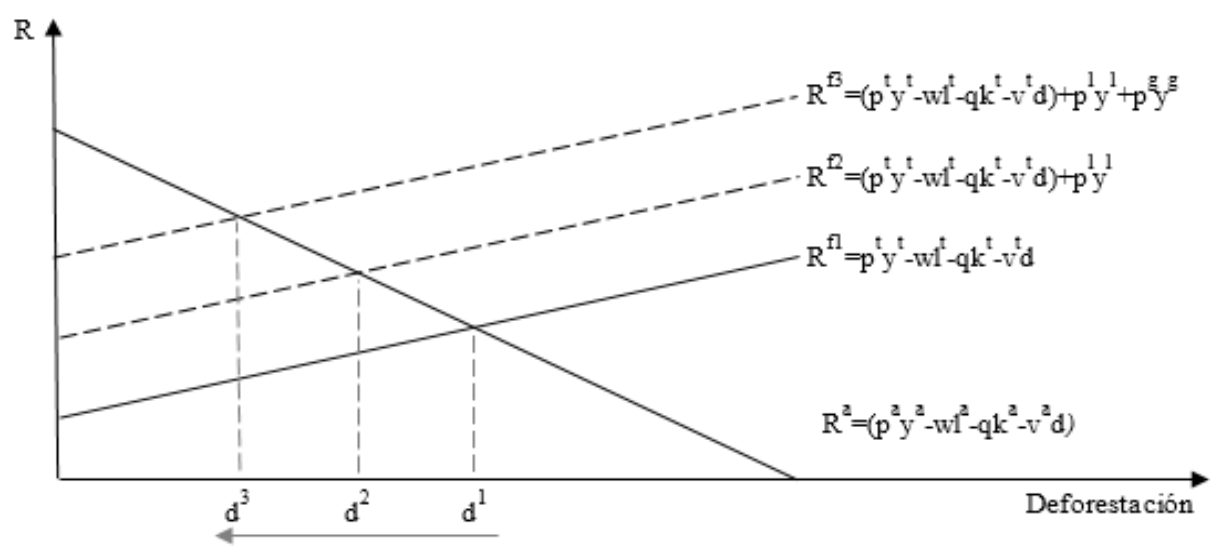

Figura 1. Interacción de las rentas del sector agrícola y forestal

Fuente: Elaboración propia adaptado de Angelsen (2010).

$\mathrm{R}^{\mathrm{a}} \quad$ es igual a los ingresos totales obtenidos de la producción ( $\left.\mathrm{p}^{\mathrm{a}} \mathrm{y}^{\mathrm{a}}\right)$ menos los costos de mano de obra $\left(\mathrm{wl}^{\mathrm{a}}\right)$, capital $\left(\mathrm{qk}^{\mathrm{a}}\right)$ y transporte $\left(\mathrm{v}^{\mathrm{a}} \mathrm{d}\right)$ (cabe precisar que la distancia está incluida en los costos de transporte). En el caso de un uso alternativo, que garantice el mantenimiento de cobertura forestal, la frontera agrícola se expande hasta la distancia donde $\mathrm{R}^{\mathrm{a}}$ es igual a cero. Los costos de transporte pueden ser demasiado altos en este punto. Por lo tanto, llegar a los mercados es demasiado costoso y se detiene la deforestación.

En el marco de la implementación de MRSE, cuanto mayor sea $\mathrm{R}^{\mathrm{f}}$ menor será la deforestación, al contrario de $\mathrm{R}^{\mathrm{a}}$, cuyo aumento incrementa la deforestación. Considerando que las rentas provenientes de las actividades forestales varían con respecto a la escala de agregación, $\mathrm{R}^{\mathrm{f}}$ puede hacerse más grande siempre que se incluyan a agentes de más niveles. A nivel local, las rentas forestales incluyen las mismas variables que las rentas agrícolas. Sin embargo, a mayor escala de agregación, como regional / nacional o global, se incluyen otros SE forestales que benefician a otros agentes que no se relacionan directamente con los bosques.

Entonces, encontrar la manera de incorporar servicios forestales locales y globales, que naturalmente no pertenecen a ningún mercado, en las decisiones de agentes productivos es el punto clave. En ese sentido, por ejemplo, Kirkby et al. (2010) encontraron que el beneficio económico de $\mathrm{SE}$ turísticos en el área natural protegida Tampopata, Madre de Dios, (US\$ 149/ ha/año) puede competir con las rentas de actividades agrícolas y ganaderas (US\$ 96/ha/año y US\$ 169/ha/año). Si esta área natural dependiera de un MRSE, el reto sería desarrollar un mecanismo que incorpore otros beneficios que se sumen a los turísticos y cuyos costos no sean restrictivos. Los MRSE aparecen como una herramienta para incluir SE, que generalmente no son comercializables, en la toma de decisiones de los agentes económicos. Hacer que $\mathrm{R}^{\mathrm{f}}$ sea lo suficientemente competitivo reduciendo puede ser la otra cara de la misma moneda (Angelsen, 2010). Sin embargo, esto no parece ser posible para un país como Perú, donde el desarrollo rural basado en la 
agricultura se considera fundamental para combatir la pobreza. Por ello el análisis de estrategias para la disminución de no es parte de este estudio.

\section{Deforestación y renta agrícola $\left(R^{a}\right)$}

Diez (10) de los 24 departamentos peruanos concentran el $95 \%$ de los 77,3 Mha de bosques en el Perú: Amazonas, Cusco, Huánuco, Junín, Madre de Dios, Loreto, Pasco, Puno, San Martín y Ucayali (Hansen et al., 2013). También acumulan casi toda la deforestación peruana en los últimos años. La Figura 2 muestra ambos aspectos haciendo una distinción entre los departamentos ubicados principalmente en la Amazonía baja y aquellos en su mayoría, en la Amazonía alta.

En la Figura 2, se evidencia que la deforestación no se explica necesariamente por la extensión de la cobertura forestal. Aunque la extensión de los bosques en la Amazonía Alta es bastante menor que la Amazonía baja, el nivel de deforestación en ambas áreas es comparable.
La ubicación de las tierras en la Amazonía alta es una explicación intuitiva para esto, pues están más cerca de las tierras altas y la costa peruana, donde se encuentran las ciudades más pobladas (incluida Lima, la capital) y por ende los mercados más importantes. La expansión agrícola es el principal impulsor de la deforestación en la Amazonía peruana (MAAP, 2015), liderada por los cultivos de café, cacao y palma aceitera (CCP).

En los últimos años, los cultivos de CCP se expandieron mucho más rápido que la agricultura agregada en los departamentos de la Amazonía peruana. Según la base de datos del Ministerio de Agricultura y Riego (MINAGRI), en los departamentos de la Amazonía alta, las áreas de CCP se expandieron $62 \%$ durante el período 2001 2014, mientras que el área total de cultivo creció 43\%. En 2014, los cultivos de CCP significaron el $30 \%$ de tal superficie. En la Amazonía baja, estos cultivos alcanzaron solo el $12 \%$ del área total de cultivo, en 2014. Sin embargo, crecieron bastante más rápido que el área total de cultivo entre 2001

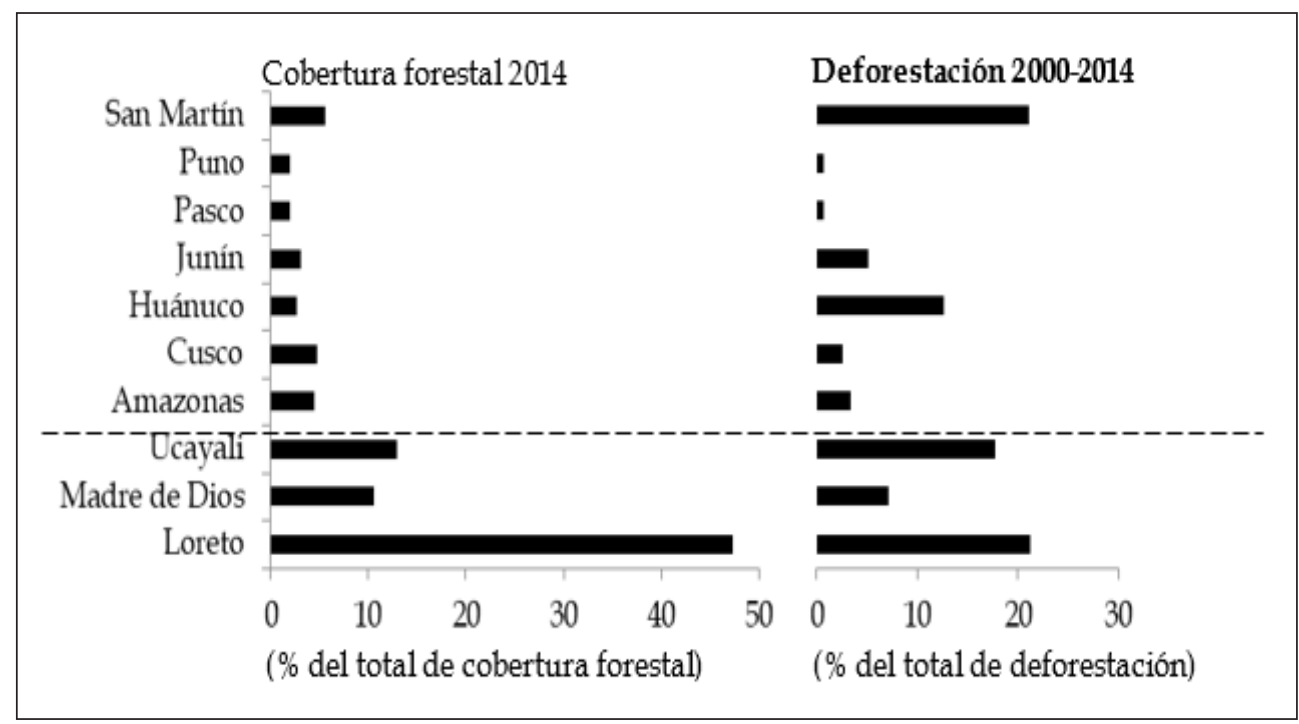

Figura 2. Departamentos de la Amazonía, cobertura forestal y deforestación

Nota: Los siete (07) primeros departamentos son clasificados parte de la Amazonía alta, mientras los restantes, como Amazonía baja. La línea discontinua separa ambos grupos.

Fuente: Hansen et al. (2013). 


\section{y 2014: 953\% frente a 33\%.}

Al contrastar la deforestación con la dinámica de los precios de los principales bienes agrícolas, utilizados como indicadores de , se aprecia una correlación positiva, en el período comprendido entre 2001 y 2014 , especialmente en el caso de los cultivos de CCP. Esto se muestra en la Figura 3, que incluye los índices de deforestación y de precios agrícolas, diferenciados por cada nivel de análisis.

A pesar del aumento de los índices de precios de los bienes agrícolas, en general, y de los índices de los cultivos de CCP, solo los últimos parecen correlacionarse junto con las cifras de deforestación. Por supuesto este resultado no es concluyente, sin embargo, tienen concordancia con la (poca) evidencia que proporcionan los estudios sobre deforestación en la Amazonía peruana.
En base a lo anterior, se puede señalar que la implementación de MRSE forestales en el Perú enfrenta un escenario adverso, pues existe evidencia de que el aumento de se correlaciona positivamente con la deforestación en la Amazonía, lo que podría sugerir un sentido de causalidad $R$ $\mathrm{R}^{\wedge} \mathrm{a}$ - deforestación. Lo anterior se hace más relevante a la luz de la expansión de los cultivos de CCP. Las Figuras anteriores sugieren que la relación - deforestación es más fuerte en la Amazonía baja que en la Amazonía alta. Sin embargo, ello no significa que la situación en la Amazonía alta sea menos preocupante.

\section{Economía ambiental y los MRSE}

La gran mayoría de SE son considerados externalidades positivas. En ese sentido, la incorporación de este tipo de bienes

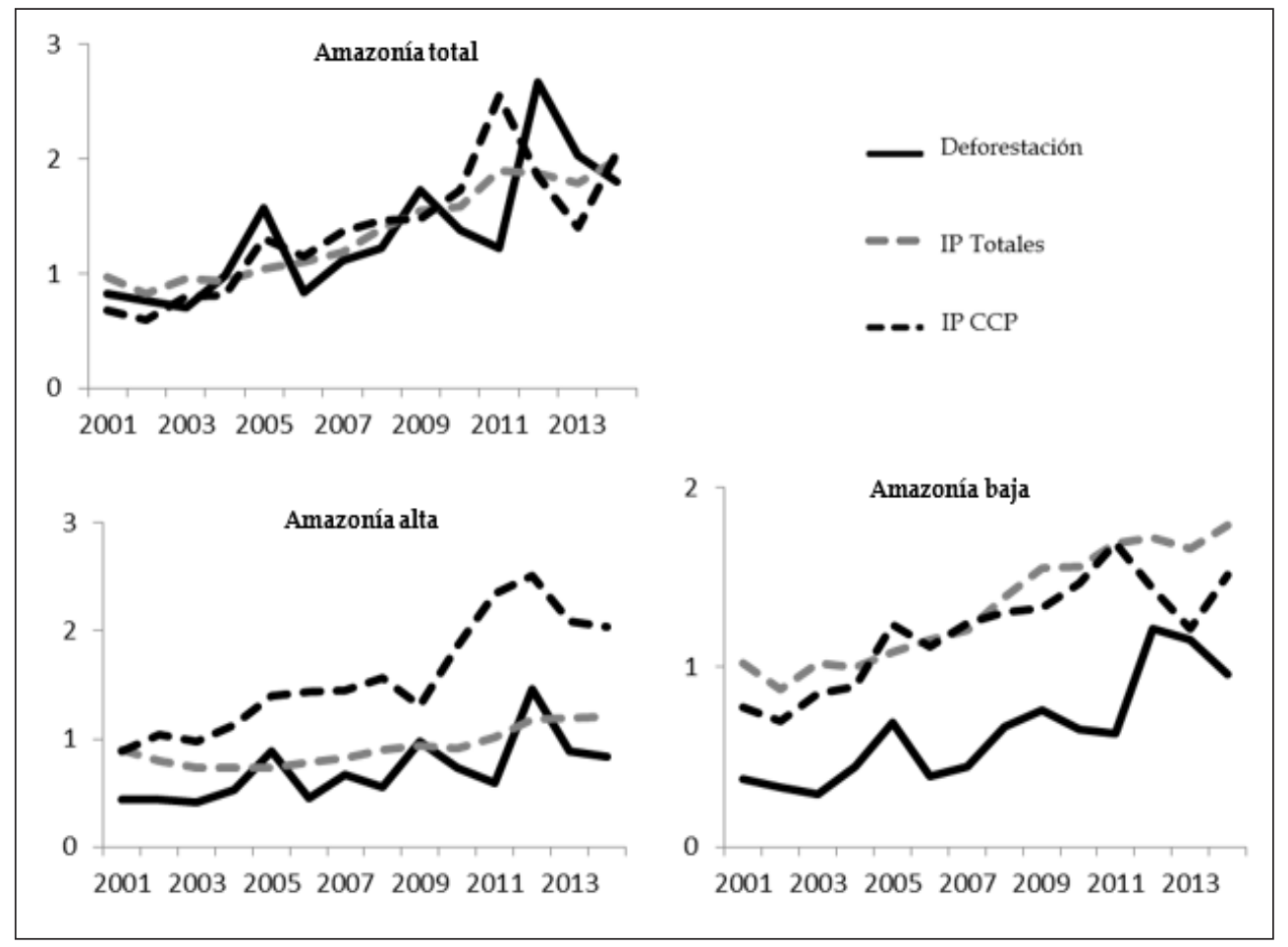

Figura 3. Tendencias de precios de los cultivos de CCP y deforestación (2000-2014) Nota: Deforestación en cientos de miles de hectáreas.

Fuente: Hansen et al. (2013), Series Históricas de Producción Agrícola - Compendio Estadístico (MINAGRI, 2016).

Julio - Diciembre 2020 
al mercado prevé el procedimiento de internalización. Una vez internalizados, los SE pasan a tener las características de bienes económicos, es decir, pasan a tener derechos de propiedad claramente definidos y precios en el mercado (Chang, 2005).

Asimismo, cabe resaltar que si el nivel óptimo de actividad económica, en términos privados - a partir de un resultado de mercado competitivo-, no coincide con una asignación eficiente, en términos sociales, es porque el mercado está generando beneficios o costos externos que conllevan a alteraciones en el bienestar de terceros; y por tanto, existen posibilidades de mejoras paretianas o ganancias en términos de eficiencia asignativa de recursos (Pearce y Turner, 1990; Muradian et al., 2010; Callan y Thomas, 2013).

La economía ambiental sostiene, básicamente, que si el ambiente, que antes era abundante, comienza a escasear (degradación de SE, contaminación antieconómica, etc.), se debe a la ausencia de reglas claras aplicables al ambiente. Entonces, si se consigue atribuir el verdadero valor a los SE, éstos podrán ser gestionados, como cualquier otro recurso económico escaso. La economía ambiental trata de crear las condiciones para que se pueda establecer el intercambio mercantil de estos bienes, allí donde antes no ocurría. Esto se conoce como el proceso de internalizar las externalidades (Pearce y Turner, 1990; Riera et al., 2016).

La identificación "exacta" de las externalidades generadas por una determinada actividad económica presenta una dificultad significativa debido a que muchas veces sus efectos presentan larga duración; es decir, permanecen en el ambiente durante largos períodos y pueden alterar el bienestar humano durante años, décadas o incluso cientos de años. En el extremo, si eso fuera así, las personas que se verían beneficiadas o afectadas por dichas externalidades pueden incluso no haber nacido aún y en ese caso no sería factible hablar de una negociación entre las partes.

En el caso en que los costos de transacción fueran superiores a los beneficios sociales, luego de la internalización de las externalidades positivas vía MRSE, la mejor asignación sería no implementar el mecanismo, pues de esa manera se evitaría una pérdida neta de bienestar. Bajo tal escenario, el nivel óptimo de externalidad sería el que incluya la falla de mercado (antes del acuerdo MRSE). Por el contrario, si los costos de transacción fueran inferiores a los beneficios sociales potenciales, se llevaría a cabo el acuerdo MRSE; y por ende se obtendría un nivel óptimo de externalidad positiva. Sin embargo, en ambos casos, los niveles de externalidad - cuando se logren internalizarlas externalidades a través de los acuerdos MRSE y cuando no- serían siempre óptimos. Esto se conoce como la teoría no falseable de la negociación coaseana (Pearce y Turner, 1990).

Los costos de transacción que se generan con mayor frecuencia en los procesos de establecimiento de acuerdos MRSE provienen principalmente de cuatro fuentes: (i) costos de monitoreo y fiscalización, (ii) costos de obtención de información relevante para negociar, (iii) costos de oportunidad de los negociadores (ingresos truncos, tiempo, etc.) y (iv) costos de certificación del cumplimiento de los acuerdos (Wunder, 2005; Riera et al., 2016).

Con relación a la concepción de externalidades como fallas de mercado; y a efectos de su corrección, se puede señalar que el paradigma de Pigou reposa sobre el papel del Estado como regulador de externalidades, mientras que Coase desestima la intervención estatal al restringir el problema del costo social exclusivamente al ámbito privado. La representación del mercado de Coase se acentúa en el derecho de propiedad, mientras que la de Pigou, se acentúa en el precio. Estas 
dos interpretaciones generan diferentes posiciones relacionadas al diseño e implementación de instrumentos de política ambiental, en general. No obstante, la eficacia y costos de dichas medidas dependerán de las instituciones del Estado. En la Tabla 2 se presentan las características y problemas de ambos enfoques de internalización de los costos y beneficios externos. para el establecimiento de MRSE distan de ser mercados reales para los servicios ecosistémicos. Cuentan con productos mal definidos, requieren constantemente de la intermediación de los gobiernos para movilizar las retribuciones desde los consumidores a los administradores de servicios ecosistémicos, y cuentan con precios preestablecidos. Esto se debe a la

Tabla 2. Características de los enfoques de Pigou y Coase en el diseño e implementación de instrumentos de política ambiental

\begin{tabular}{lll}
\hline Características & Pigou & Coase \\
\hline Principio & Contaminador (beneficiario) -pagador & Derechos de propiedad \\
Modelo & Centralizado & Descentralizado \\
Estrategia & Intervención del Estado & Medidas de libre mercado \\
Políticas ambientales & Licencias, impuestos & Negociación privada previa \\
Principal ventaja & $\begin{array}{l}\text { Efectividad respecto de los objetivos } \\
\text { de política ambiental }\end{array}$ & Bajos costos para el Estado \\
\hline Fuente: Elaboración propia adaptado de Chang $(2005)$ &
\end{tabular}

Fuente: Elaboración propia adaptado de Chang (2005).

\section{Economía institucional y los MRSE: problemas latentes}

El establecimiento de los MRSE requiere estructuras de gobernanza específicas para apoyar la creación de mercados u otros instrumentos de asignación de recursos. Esta es una perspectiva que carece el desarrollo actual sobre el establecimiento de MRSE, ya que "no enfatiza los problemas específicos involucrados al crear un mercado para servicios ambientales, específicamente cómo los costos de transacción influyen en el formato de los pagos" (Vatn, 2010). Además, excluye otras estructuras de gobernanza, diferentes del mercado y que permiten la asignación de recursos: jerarquías (Williamson, 2000) y manejo comunitario (Ostrom, 1990).

Sin embargo, por sus características, el manejo de SE ajusta en muchas ocasiones a estructuras de gobernanza distintas al mercado o que los trascienden. Corbera et al. (2007) señalan que las estructuras existencia de costos de transacción positivos, que justifica una posible intervención en la economía por parte del Estado y del sistema jurídico, algo que se desprende del teorema de Coase (Caballero, 2002).

En este sentido, antes que establecer estructuras de manejo predeterminadas, es necesario elegir aquellas reglas legales, procedimientos y estructuras administrativas que reduzcan los costos de establecer los MRSE. Para elegir este tipo de instituciones hay que analizar los costos de transacción y comparar soluciones institucionales alternativas para el manejo de los SE y asegurar de su provisión al mismo tiempo que se eviten costos de establecimiento prohibitivos (Coase, 1994; Vatn, 2010).

La implementación de las estructuras de gobernanza que buscan asemejarse al mercado implica la generación de costos de transacción significativos. Esto involucra el establecimiento de normas y de derechos de propiedad sobre los 
recursos en cuestión. Es posible que estos contravengan en instituciones previas, incrementando los costos de transacción asociados. "Muchos agentes pueden estar involucrados. La confianza puede ser baja y la creación de confianza se hace necesaria. Cuando se hace un contrato, es necesario verificar si lo que se contrata también es efectivamente entregado" (Vatn, 2010, p. 1248). Todo esto implica costos, que no son contabilizados como costos directos de asegurar la provisión de los SE. Vatn (2010), señala que otras estructuras de gobernanza diferentes al mercado, tales como las jerarquías o la gestión comunitaria, pueden reducir los costos de transacción de manera significativa.

La mercantilización, que es necesaria para establecer mercados de SE, no siempre es posible debido a que los SE no pueden aislarse completamente de otros servicios con potencial de mercantilización porque provienen de la interacción compleja de los procesos ecológicos de los ecosistemas. Además, es posible la aparición de free riders, ya que no es posible incluir a todos los beneficiarios en los esquemas de MRSE (Van Hecken y Bastiaensen, 2010). Por ejemplo, los contribuyentes que pagan por la protección de cobertura boscosa dentro de un MRSE también pagan por proveer un secuestro de carbono globalmente beneficioso. Esto hace deseable la inclusión del mayor número posible de agentes en las transacciones que involucran diversos SE. Sin embargo, dado que la separación completa de un SE, de otros servicios, no es posible; y los costos de transacción de incluir más agentes pueden ser significativos, parece lógico aceptar cierto nivel de free riders.

En este sentido, es indispensable hacer un análisis de las potenciales estructuras de gobernanza para manejar los MRSE, así como los costos implicados en el diseño e implementación de cada una de estas estructuras. La valoración y fijación de precios de SE y las estructuras de gobernanza necesarias para lo anterior son dos caras de la misma moneda en lo que respecta a la conservación forestal, que parecen estar separadas en el entendimiento común (economía ambiental) de los MRSE, más aún cuando los fundamentos teóricos de tales mecanismos de mercado no permiten que se materialice en el análisis la cuestión institucional fundamental, pero latente. No obstante, cabe precisar que los esquemas de MRSE implican diferentes costos de transacción con respecto a la instalación de estructuras de gobernanza como alternativa a este tipo de instrumentos de mercado (Wunder, 2005; Riera et al., 2016).

\section{Economía ambiental e institucional: la importancia de los costos de transacción}

La definición común de los MRSE es demasiado limitada para incluir todos los aspectos vinculados a su implementación. Estos, generalmente involucran poblaciones rurales donde en la gran mayoría de casos existe situación de pobreza y/o pobreza extrema e instituciones que no necesariamente son compatibles con los MRSE. En ese marco se hace necesario el diseño de la estructura institucional pues si bien es cierto existe una estructura básica con la ley y el reglamento de los MRSE, las organizaciones de la sociedad civil son todavía débiles en el conocimiento del funcionamiento y la gestión de estos acuerdos, más aún cuando los aspectos institucionales, relacionados a costos de transacción - significativos-, permanecen fuera del concepto de MRSE generalmente utilizado.

Los fundamentos teóricos de la economía ambiental, respecto de los MRSE, llevan a centrarse casi exclusivamente en los 
mercados, lo que restringe la búsqueda de otras estructuras de gobernanza alternativas. No obstante, debido a que la mayoría de los SE presentan características de bienes públicos y son no separables, los mercados probablemente no aparecerán. Según Vatn (2010), las estructuras jerárquicas con liderazgo estatal o gestión comunitaria pueden conducir a resultados más deseables pues el enfoque de mercado implica costos de transacción significativos en la práctica.

En base al modelo de competencia de rentas presentado, se puede señalar que el Perú tiene que superar grandes desafíos para utilizar MRSE adecuadamente como estrategia para la conservación de los bosques en la Amazonía. La expansión agrícola y el aumento de, específicamente de los cultivos de CCP, hacen urgente el incremento del valor de, sea incluyendo otros SE o buscando más contribuyentes.

Sin embargo, la atención no debe estar centrada solamente en y . Como se revisó, la implementación de los MRSE depende fuertemente de los costos de transacción. Los modelos de rentas no contemplan estos costos. Por lo que debe buscarse la forma de incorporarlos al proceso de toma de decisión. Quintero y Pareja (2015), al identificar y analizar cuellos de botella en la implementación de los esquemas de MRSEH en el Perú, resaltan aspectos técnicos que cuestionan la estimación de la compensación monetaria y la definición del (de los) SE a comercializar; sin embargo, los aspectos institucionales y financieros aparecen como los más problemáticos. Los aspectos financieros también pueden considerarse institucionales, estas se refieren principalmente a flexibilizar las restricciones y ajustar las normas para simplificar la administración de los fondos recaudados.

Mantener flujos de financiamiento constantes y potenciar la gobernanza sobre los fondos recaudados es el principal problema de los MRSE. El hecho de que los fondos recaudados se conviertan en fondos públicos es una limitación para los actores no públicos cuando se desean establecer estructuras de gobernanza. Ellos no tienen voz para decidir cómo se deben ejecutar los gastos de los referidos fondos, dado que la gestión de la inversión pública es una prerrogativa exclusiva de los organismos públicos. Además, los fondos provenientes de actores privados u ONG no son fáciles de administrar conjuntamente con el gasto público, a menos que se conviertan en fondos públicos.

Por todo lo expuesto, es comprensible que la implementación de MRSE presente grandes desafíos en la Amazonía, pues en ese contexto se requerirán esquemas de MRSE para capturar mucho más valor de los SE forestales, además de los de provisión. Los arreglos institucionales necesarios para establecer estructuras de gobernanza y facilitar la administración de fondos recaudados son la otra parte del problema, aunque este último aspecto no se relaciona exclusivamente con la región amazónica ni con SE forestales solamente.

\section{Conclusiones}

El presente estudio evidencia que existe aún espacio de discusión respecto de la comprensión común de los MRSE y los factores que limitan la implementación eficiente de estos instrumentos de mercado. Esto ocurre especialmente en el caso de políticas de deforestación —más aún si se considera un modelo de competencia de rentas por el uso de tierras tal como se analizó en el ámbito de la Amazonía peruana-, pues la definición común de los MRSE se basa, operacionalmente, en las fuentes de los SE al no poder aislar el SE específico del total de SE que proveen los ecosistemas.

Desde la economía ambiental, la estructura de este mecanismo de mercado reconoce la importancia de los costos de transacción en 
el proceso de compraventa de externalidades positivas (internalización). No obstante, se reconoce que muchas veces este aspecto es asumido por agentes externos al mercado. La economía institucional extiende este análisis al enfatizar sobre las serias limitaciones para incluir todos los aspectos externos del mercado en el establecimiento de esquemas MRSE. Lamentablemente, incluso cuando las restricciones institucionales se reconocen adecuadamente, no son una parte estructural de los MRSE. En ese sentido, una definición de MRSE sustentada también en la teoría institucional podría ser útil al abordar la conservación forestal de una manera amplia, abriendo incluso el uso de estructuras de gobernanza alternativas al mercado para el establecimiento de estos mecanismos.

\section{Literatura citada}

Angelsen, A 2010. Policies for reduced deforestation and their impact on agricultural production. PNAS 107(46):19639-19644.

Bruner, A.G.; Gullison, R.E.; Rice, R.E.; Da Fonseca, G.A. 2001. Effectiveness of parks in protecting tropical biodiversity. Science, 291(5501): 125-128.

Caballero, M.G. 2002. Economía ambiental: perspectiva institucional.

Callan, J.; Thomas, J. 2013. Modeling Market Failure. Environmental Economics and Management: Theory, Policy and Applications. (52-75). Delhi, India. Cengage Learning.

Chang, M.Y. 2005. La economía ambiental. Sustentabilidad, 165-178.

Coase, R. 1994: La empresa, el mercado y la ley. Alianza Economía.

Corbera, E.; Kosoy, N.; Martínez-Tuna, M. 2007. Equity implications of marketing ecosystem services in protected areas and rural communities: Case studies from Meso-America. Global Environmental Change, 17:365-380.

Curtis, P.G.; Slay, C.M.; Harris, N.L.; Tyukavina, A.; Hansen, M. C. 2018. Classifying drivers of global forest loss. Science, 361(6407): 1108-1111.

Finer, M.; Mamani, N. 2020. MAAP Synthesis: 2019 Amazon Deforestation Trends and Hotspots. MAAP Synthesis, 4.

Finer, M.; Mamani, N.; García, R.; Novoa, S. 2018. Hotspots de Deforestación en la Amazonía Peruana, 2017.

Finer, M.; Mamani, N. 2018. Deforestación en la Amazonía Andina (Tendencias, Hotspots, Drivers). MAAP Sintesis, 3.

Goldman, R.L.; Tallis, H. 2009. A Critical Analysis of Ecosystem Services as a Tool in Conservation Projects: The Possible Perils, the Promises, and the Partnerships. In Ostfeld R.S., W. H. Schlesinger (Editores). Annals of the New York Academy of Sciences: The Year in Ecology and Conservation Biology. Nueva York, Wiley-Blackwell Publishing.

Global Forest Watch 2020 Disponible en https://www.globalforestwatch.org/ dashboards/country/PER

Hajek, F.; Martínez de Anguita, P. 2012. ¿Gratis? Los servicios de la naturaleza y cómo sostenerlos en el Perú. Servicios Ecosistémicos Perú. Lima-Perú.

Hanley, N.; Shogren, J.; White, B. 2001. Introduction to Environmental Economics. Oxford, UK, Oxford University. 299 p.

Hansen, M.; Potapov, P.; Moore, R.; Hancher, M.; Turubanova, S.; Tyukavina, A.; Thau, D. et al. 2013. Hansen/UMD/ Google/USGS/NASA Tree Cover Loss and Gain Area. University 
of Maryland, Google, USGS, and NASA.

Kalamandeen, M.; Gloor, E.; Mitchard, E.; Quincey, D.; Ziv, G.; Spracklen, D.; Galbraith, D. 2018. Pervasive rise of small-scale deforestation in Amazonia. Scientific reports, 8(1): 1-10.

Kirkby, C.A.; Giudice-Granados, R.; Day, B.; Turner, K.; Velarde-Andrade, L. M.; Dueñas-Dueñas, A.; Douglas, W.Y. 2010. The market triumph of ecotourism: an economic investigation of the private and social benefits of competing land uses in the Peruvian Amazon. PloS one, 5(9):e13015.

Lucich, I.M.; Villena, M.G.; Quinteros, M. J. 2015. Transportation costs, agricultural expansion and tropical deforestation: Theory and evidence from Peru. International Journal of Agriculture and Natural Resources, 42(2),:153-169.

La Rosa Salazar, M. 2016. Determinantes de la deforestación en el Perú: evidencia de un panel de datos de diez departamentos para el período 2001- 2012. Universidad Nacional Agraria La Molina, Lima, Perú.

Llerena, C.; Yalle, S. 2014. Los servicios ecosistémicos en el Perú. Xilema, 27: 62-75.

MAAP [Monitoring of the Andean Amazon Project]. 2015. Patrones y drivers de la deforestación en la Amazonía peruana. Disponible en: www. maaproject.org/2015/maap-sintesis1/

MEA [Millenium Ecosystem Assessment]. 2005. Ecosystems and Human Well-being: synthesis. Island Press, Washington DC. Disponible en: www.millenniumassessment.org/ documents/document.356.aspx.pdf
MINAG [Ministerio de Agricultura]. 2011. Ley 29763 - Ley Forestal y de Fauna Silvestre. Disponible en: www. actualidadambiental.pe/wp-content/ uploads/2013/12/Ley-Forestal-y-deFauna-Silvestre-29763.pdf

MINAG y MINAM. 2011. El Perú de los bosques. Disponible en: www. cdam.minam.gob.pe/novedades/ elperudelosbosques2011.pdf

MINAM. 2010. Política Nacional del Ambiente. Retrieved April 12, 2017. Disponible en: www.minam.gob. pe/wpcontent/uploads/2013/08/ Pol\%C3\%ADtica-Nacional-delAmbiente.pdf

MINAM. 2014. Borrador de la Estrategia Nacional de Cambio Climático. Disponible en: www.minam.gob. pe/wp18content/uploads/2014/07/ Estrategia-Nacional-ante-elCambio-Climatico_ENCC.pdf

MINAM. 2016. Estrategia Nacional sobre Bosques y Cambio Climático. Disponible en: www. bosques.gob.pe/archivo/ff3f54 OCLIMATICO2016 ok.pdf

MINAM. 2016a. Decreto Supremo Nº092016. Disponible en: busquedas. elperuano.pe/download/url/ aprueban-reglamento-de-la-leyn-30215-ley-de-mecanismos-dedecreto-supremo-n-009-2016minam-1407244-4

Muradian, R.; Kumar, P. 2009. Payment for ecosystem services and valuation: challenges and research gaps. Payment for ecosystem services, 1-16.

Muradian, R.; Corbera, E.; Pascual, U.; Kosoy, N.; May, P.H. 2010. Reconciling theory and practice: An alternative conceptual framework for understanding payments for environmental services. Ecological 
economics, 69(6): 1202-1208.

Ostrom, E. 1990. Governing the commons: The evolution of institutions for collective action. Cambridge university press.

Pearce, D.; Turner, K. 1990. El logro de la contaminación óptima a través del Mercado. Economía de los Recursos Naturales y del Medio Ambiente: 103-113. Madrid, España. CELESTE EDICIONES.

Pulgar-Vidal, M. 2016. El Acuerdo de París: el largo proceso hacia el éxito. Rol, retos y oportunidades para el Perú. Lima, Perú: Ministerio del Ambiente. pp. 73. Disponible en: http://www2.congreso.gob.pe/sicr/ biblioteca/Biblio_con.nsf/999a45 $849237 \mathrm{~d} 86 \mathrm{c} 052577920082 \mathrm{c} 0 \mathrm{c} 3 /$ FILE/333.716-P95.PDF

Quintero, M.; Pareja, P. 2015. Estado de Avance y Cuellos de Botella de los Mecanismos de Retribución por Servicios Ecosistémicos Hidrológicos en Perú. Centro Internacional de Agricultura Tropical (CIAT), Cali, Colombia. 40 p.

Riera, P.; García, D.; Kristrom, B.; Brannlund, R. 2016. Valoración ambiental (I). Métodos de preferencias reveladas. Manual de economía ambiental y de los recursos naturales. (111116). Madrid, España. Ediciones Paraninfo, S.A

Smith, K.R. 2006. Health impacts of household fuelwood use in developing countries. UNASYLVAFAO-, 57(2): 41.

Series Históricas de Producción AgrícolaCompendio Estadístico. MINAGRI, 2016. Disponible en: www. frenteweb.minagri.gob.pe/sisca/

TEEB - The Economics of Ecosystem and Biodiversity. 2010. Chapter
2: Biodiversity, ecosystems and ecosystem services. Disponible en: www.img.teebweb.org/wp-content/ uploads/2013/04/D0-Chapter2-Biodiversity ecosystems-andecosystem-services.pdf

Van Hecken, G.; Bastiaensen, J. 2010. Payments for ecosystem services: justified or not? A political view. Environmental Science and Policy, 13.

Vatn, A. 2010. An institutional analysis of payments for ecosystem services. Ecological Economics, 69: 12451252 .

Vijay, V.; Reid, C. D.; Finer, M.; Jenkins, C. N.; Pimm, S. L. 2018. Deforestation risks posed by oil palm expansion in the Peruvian Amazon. Environmental Research Letters, 13(11): 114010.

Wolcott, R. 2006. Prospects for Ecosystem Services in the Future Agricultural Economy: Reflections of a Policy Hand, American Journal of Agricultural Economics, 88(5):11811183.

Williamson, O.E. 2000. Taking stock, looking ahead. journal of Economic Literature, 38(3): 595-613.

Wunder, S. 2005. Payments for environmental services: some nuts and bolts. CIFOR, occasional paper $\mathrm{N}^{\circ} 42$.

Wunder, S.; Engel, S.; Pagiola, S. 2008. Taking stock: A comparative analysis of payments for ecosystem services in developed and developing countries. Ecological Economics, 65: 834-852.

Zegarra, E.; Gayoso, J.P. 2015. Cambios en la agricultura y deforestación en la selva peruana: análisis basado en el IV Censo Agropecuario. 